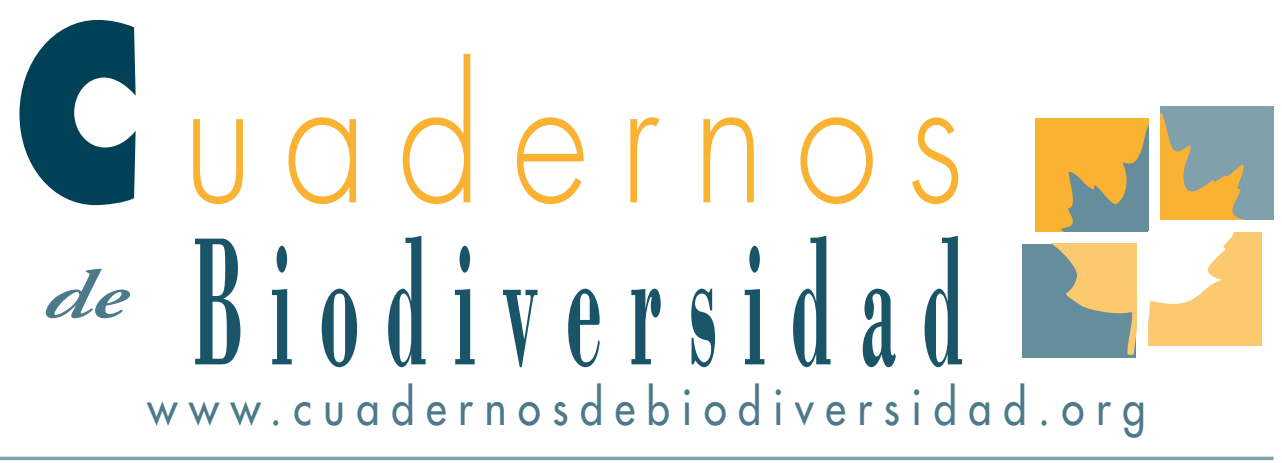

\title{
Primer registro de Aedes albopictus (Skuse, 1894) en la mancomunidad de l'Alacantí. Acciones para evitar su dispersión
}

\section{Rubén Bueno Mari ${ }^{1 *}$, José Andrés Lluch Escribano ${ }^{2}$ \& Braulio Gambin Molina ${ }^{2}$}

i Departamento de Investigación y Desarrollo (I+D), Laboratorios LoKímica

2Mancomunidad de l'Alacantí

*Correspondencia: Ruben.Bueno@UV.es; RbUeno@LOKimicA.ES

\section{ABSTRAT}

This paper details the first data of the presence of Asian tiger mosquito, Aedes albopictus (Skuse, 1894), in the Mancomunidad de l'Alacantí (Southeastern Spain). The implications of the establishment of the species in this region and control measures needed to apply are also briefly discussed in the text.

\section{KEY WORDS}

Aedes albopictus, mosquito control, vector management, medical entomology, entomological surveillance, public health.

\section{INTRODUCCIÓN}

Aedes albopictus (Skuse, 1894), comúnmente conocido como "mosquito tigre", es una especie exótica invasora de hábitats humanos y de elevada trascendencia para la salud pública al ser un potencial vector de arbovirosis de primera magnitud sanitaria como el dengue o la fiebre amarilla (Bueno Marí \& Jiménez Peydró, 2012). Se detectó por primera vez en España en la localidad barcelonesa de San Cugat del Vallès en el año 2004 (Aranda et al., 2006) y en los últimos años ha ido expandiéndose hacia el sur por la franja litoral mediterránea. Actualmente la especie está ampliamente distribuida en las tres provincias costeras catalanas, y se ha establecido de 
manera puntual e irregular en las provincias de Castellón, Valencia, Alicante, Murcia y Baleares (Roiz et al., 2007; Bueno Marí et al., 2009, 2012, 2013; Delacour et al., 2010; Collantes \& Delgado, 2011; Miquel et al., 2013).

Dado que $A$. albopictus es una especie con un rango de vuelo relativamente bajo, raramente superior a los 500 metros (Hawley, 1988), es más que probable que estas recientes colonizaciones hayan tenido lugar a partir del desplazamiento involuntario o accidental de adultos en el interior de vehículos, tal y como ya ha sido evidenciado en otros países europeos (Flacio et al., 2004). Sin embargo, tampoco pueden descartarse episodios de dispersión a larga distancia de formas inmaduras también constatados en otros países, como por ejemplo la diseminación de huevos en neumáticos usados (Reiter \& Sprenger, 1987) y larvas en productos de jardinería asociados al bambú (Madon et al., 2002).

\section{MATERIAL Y MÉTODOS}

En el año 2013, se instauró un programa de vigilancia entomológica en los municipios pertenecientes a la Mancomunidad de l'Alacantí con el objetivo de detectar rápidamente el establecimiento de $A$. albopictus y poder así comenzar a adoptar las medidas de control integral oportunas. Dicho sistema de vigilancia se basó en dos estrategias de búsqueda diferentes:

1) Detección pasiva mediante la instalación de trampas de captura de mosquitos tipo BGSentinel (Fig. 1) en enclaves estratégicos de relevancia para el posible asentamiento de la especie.

2) Detección activa mediante inspecciones de posibles criaderos de la especie, a partir de un proceso de filtrado de avisos o incidencias procedentes de la ciudadanía, cuyas descripciones coincidieran con la biología y comportamientos hematofágicos típicos del mosquito tigre.

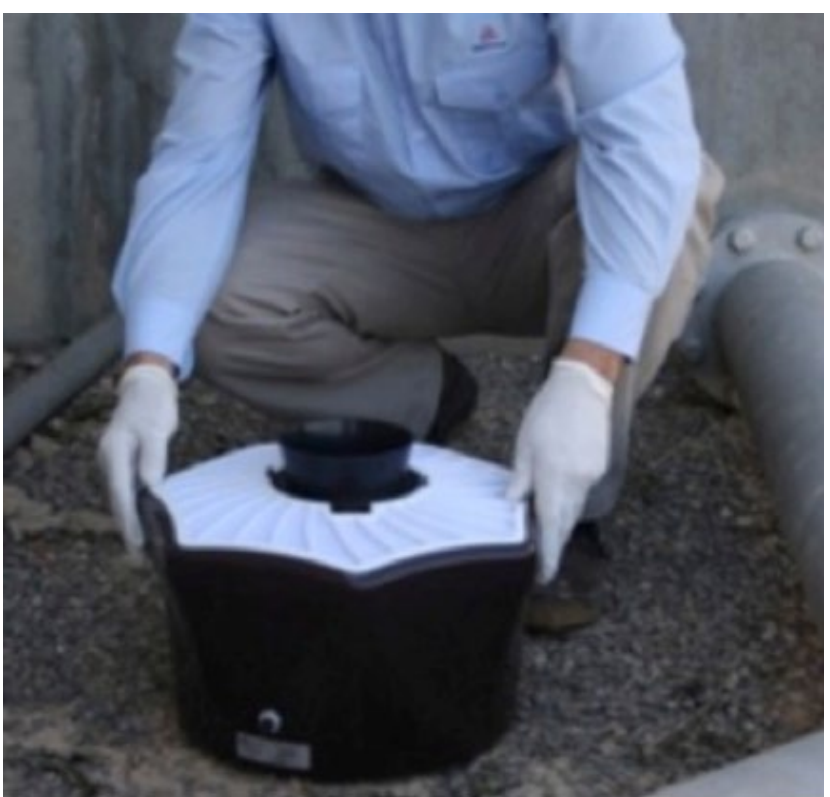

Figura 1: Instalación de trampa BG-Sentinel para la detección del mosquito tigre

\section{RESULTADOS}

Pese a que el material entomológico analizado en las trampas de captura no permitió la identificación del mosquito tigre, ni ninguna otra de las especies invasoras de mosquitos que están detectándose en Europa en los últimos años como Aedes aegypti (Linnaeus, 1762), Aedes atropalpus (Coquillett, 1902), Aedes japonicus (Theobald, 1901), Aedes koreicus (Edwards, 1917) o Aedes triseriatus (Say, 1823), una de las inspecciones realizadas en agosto de 2013 permitió la determinación de ejemplares larvarios y adultos de $A$. albopictus en una zona residencial de viviendas unifamiliares de la localidad de El Campello (38 26’ 40” N / 0²1' 50” O) (Fig. 2).

Se recolectaron decenas de ejemplares larvarios en recipientes domésticos que acumulaban agua para fines de jardinería (Figs. 3 y 4) en diversas viviendas unifamiliares contiguas. También, mediante aspiradores mecánicos, se capturaron hembras en las inmediaciones de los puntos de cría y sobre hospedadores humanos con intención de picar. Todo el 

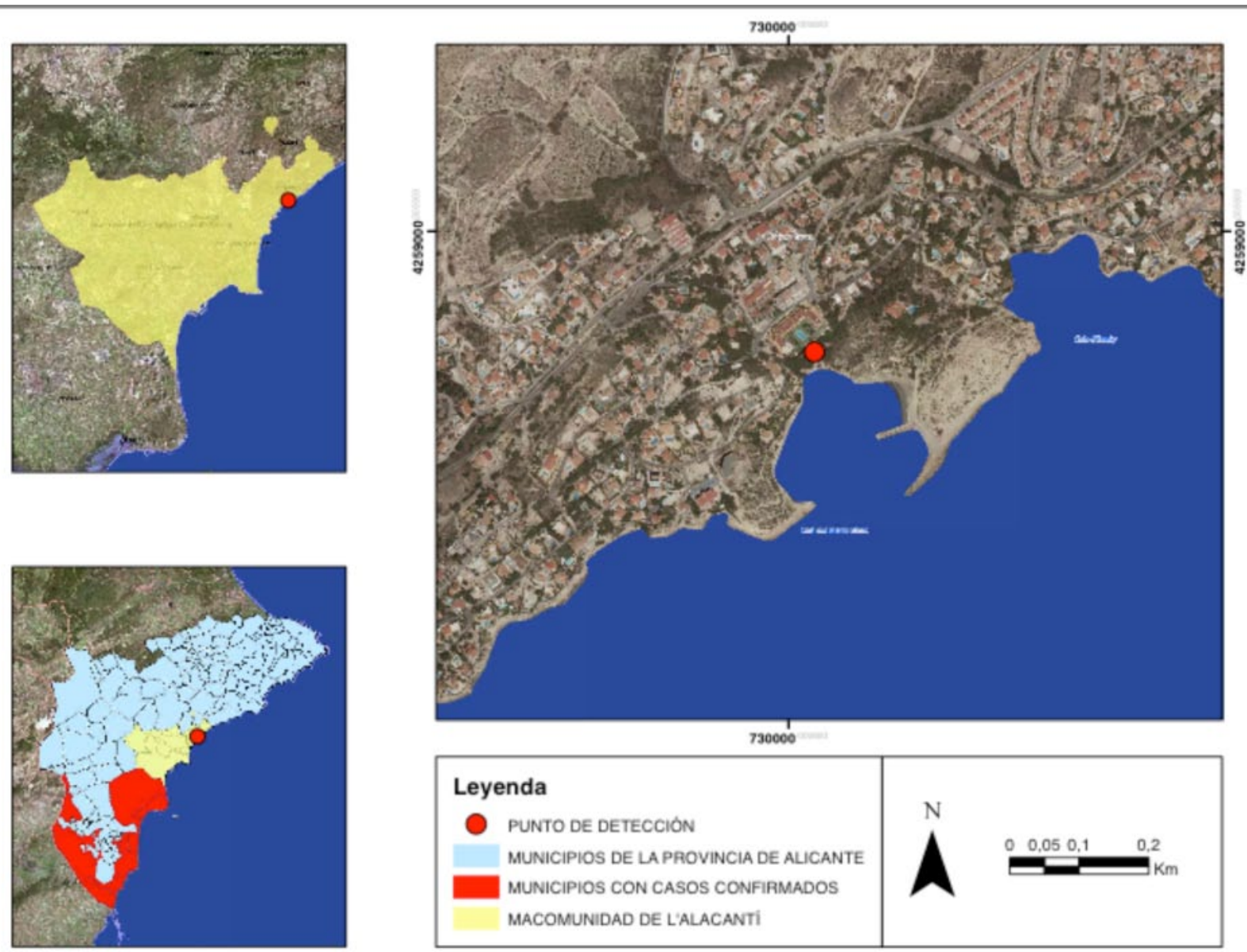

Figura 2. Situación geográfica del primer hallazgo del mosquito tigre en la Mancomunidad de l'Alacantí (Alicante, España).

material entomológico recolectado fue transportado al laboratorio, donde se determinó bajo observación de lupa binocular y en apoyo de las claves de Schaffner et al. (2001), que todos los ejemplares pertenecían a la especie $A$. albopictus.

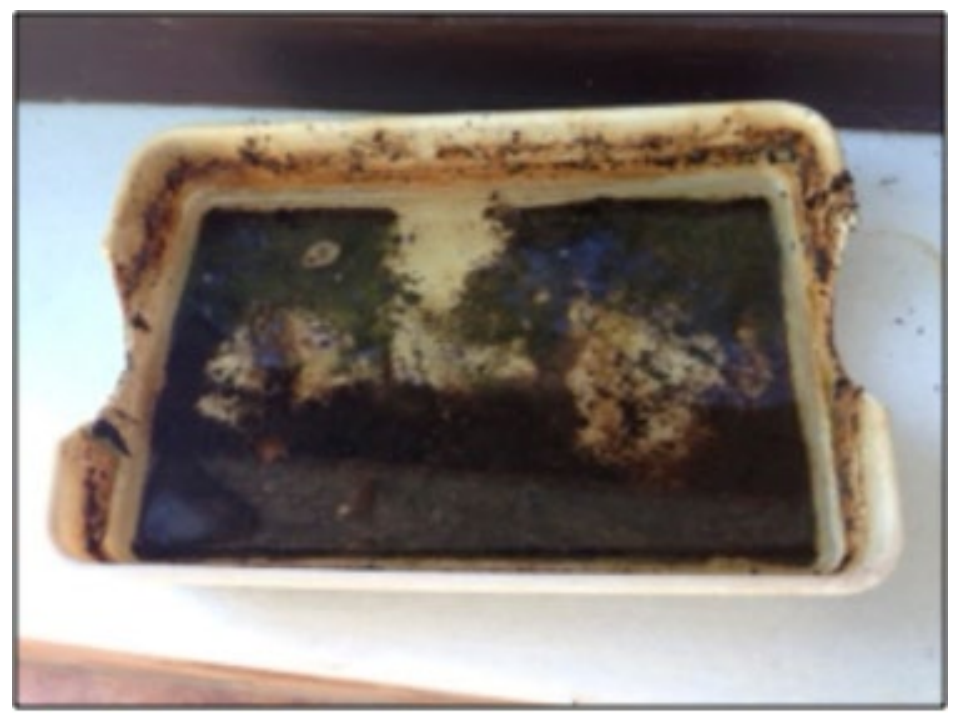

Figura 3. Recipiente donde se detectaron larvas del mosquito tigre. 


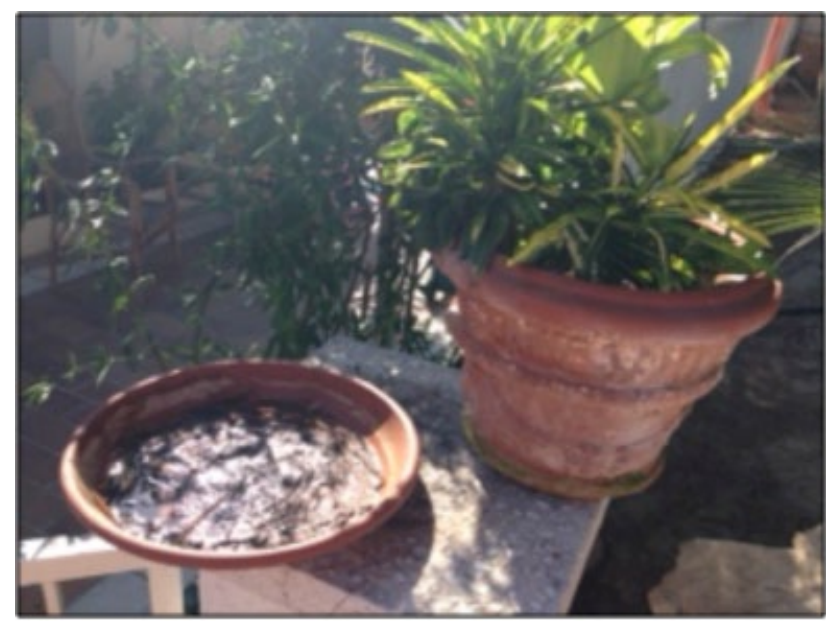

Figura 4. También se colectaron estadíos preimaginales del mosquito tigre en diversos platos que recogen excedentes del agua de riego de macetas.

\section{DISCUSIÓN}

El establecimiento del mosquito tigre en la Mancomunidad de l'Alacantí puede tener una serie de implicaciones en la velocidad de expansión de la especie por la provincia. La Mancomunidad destaca por presentar una elevada densidad de población (donde se congregan cerca de una cuarta parte de los habitantes de la provincia) y excelentes infraestructuras de comunicación terrestre con el resto de municipios. Esta situación puede favorecer una rápida diseminación de la especie asociada al desplazamiento accidental de adultos en el interior de vehículos. De hecho, una de las principales causas de la rápida expansión del mosquito tigre en Catalunya, en relación a lo sucedido en otros lugares, es precisamente que el punto de origen de su detección fue en el Área Metropolitana de Barcelona, donde existe una gran densidad poblacional y un importante tráfico rodado diario.

Cabe destacar que en la Mancomunidad de l'Alacantí, esta especie solo ha sido detectada en el ámbito de la propiedad privada, no evidenciándose hasta el momento la presencia de puntos de cría en biotopos de titularidad pública como imbornales o fuentes ornamentales. Esta situación dificulta enormemente el control poblacional desde la administración pública, ya que todo el peso de las acciones preventivas y correctivas a adoptar recae exclusivamente en los propietarios. De cara al futuro, la estrategia de control integrado a abordar pasa por el desarrollo de, al menos, las siguientes acciones:

- Intensificar la vigilancia entomológica en posibles nuevas áreas de colonización para detectar con rapidez la llegada de la especie y comenzar con las acciones correctivas pertinentes en situaciones de baja densidad poblacional.

- Implementar proyectos divulgativos y educativos para concienciar a la ciudadanía en que su papel es clave para evitar la proliferación de la especie en el ámbito privado.

- Establecer programas de control larvario sistemáticos en puntos de riesgo de titularidad pública.

- Desarrollar Sistemas de Información Geográfica (SIG) que ayuden en la interpretación de los resultados de las campañas de control poblacional y del seguimiento fenológico de la especie en un área tan propicia a nivel climatológico como es la Mancomunidad de l'Alacantí.

\section{AGRADECIMIENTOS}

Se agradece la colaboración y asesoramiento prestado por los doctores Ricardo Jiménez (Universitat de València), Eduardo Galante y Ma Ángeles Marcos (CIBIO, Universidad de Alicante), y Ana Pineda (Wageningen University, Holanda).

\section{REFERENCIAS}

Aranda, C., Eritja, R. \& Roiz, D. 2006. First record and establishment of the mosquito Aedes albopicus in Spain. Med. Vet. Entomol. 20: 150-152.

Bueno Marí, R. \& Jiménez Peydró, R. 2012. Implicaciones sanitarias del establecimiento y expansión en España del mosquito Aedes albopictus. Rev. Esp. Salud Pública. 86: 319-330. 
Bueno Marí, R., Chordá Olmos, F.A., Bernués Bañeres, A. \& Jiménez Peydró, R. (2010). Detección de Aedes albopictus (Skuse, 1894) en Torrevieja (Alicante, España). Bol. Asoc. Esp. Entomol. 33: 529-532.

Bueno Marí, R., García Mújica, P., Rico Miralles, J. \& Agulló Ronco, A. 2012. Nuevos datos sobre el proceso de expansión de Aedes albopictus (Skuse, 1894) (Diptera, Culicidae) por el sureste de la Península Ibérica: hallazgo de la especie en Mazarrón (Murcia, Espańa). Bol. S.E.A. 51: 307-309.

Bueno Marí, R., Bernués Bañeres, A., Muñoz Rodríguez, M. \& Jiménez Peydró, R. 2013. Primera cita de Aedes albopictus (Skuse, 1894) en la provincia de Valencia (Diptera, Culicidae). Bol. Asoc. Esp. Entomol. 37: 375-378.

Collantes, F. \& Delgado, J.A. 2011. Primera cita de Aedes (Stegomyia) albopictus (Skuse, 1894) en la Región de Murcia. An. Biol. 33,99-101.

Delacour Estrella, S., Bravo Minguet, D., Alarcón Elbal, P.M., Bengo, M., Casanova, A.,Melero Alcíbar, R., Pinal, R., Ruiz Arrondo, I., Molina, R. \& Lucientes, J. 2010. Detección de Aedes (Stegomyia) albopictus (Skuse, 1894) (Diptera: Culicidae) en Benicàssim. Primera cita para la provincia de Castellón (España). Bol. S.E.A. 47: 440.

Flacio, E., Lüthy, P., Pattochi, N., Guidotti, F., Tonolla, M. \& Peduzzi, R. 2004. Primo ritrovamento di Aedes albopictus in Svizzera. Bollettino STSN. 92: 141-142.
Madon, M.B., Mulla, M.S., Shaw, M.W., Kul, S. \& Hazelrigg, J.E. 2002. Introduction of Aedes albopictus (Skuse) in southern California and potential for its establishment. J. Vector Ecol. 27: 149-154.

Hawley, W. 1988. The biology of Aedes albopictus. J. Am. Mosq. Control. Assoc. 1: 1-39.

Miquel, M., del Río, R., Borràs, D., Barceló, C., Paredes Esquivel, C., Lucientes, J. \& Miranda, M.A. 2013. First detection of Aedes albopictus (Diptera: Culicidae) in the Balearic Islands (Spain) and assessment of its establishment according to the ECDC guidelines. J. Eur. Mosq. Control Assoc. 31: 8-11.

Reiter, P. \& Sprenger, D. 1987. The used tire trade: a mechanism for the worldwide dispersal of container breeding mosquitoes. J. Am. Mosq. Control. Assoc. 3: 494-501.

Roiz, D., Eritja, R., Melero Alcíbar, R., Molina, R., Marqués, E., Ruiz, S., Escosa, R., Aranda, C. \& Lucientes, J. 2007. Distribución de Aedes (Stegomyia) albopictus (Skuse, 1894) (Diptera, Culicidae) en España. Bol. S.E.A. 40: 523-526.

Schaffner, F., Angel, G., Geoffroy, B., Hervy, J.O. \& Rhaeim, A. 2001. The mosquitoes of Europe/Les moustiques d'Europe (programa de ordenador). Montpellier, France: IRD Éditions and EID Méditerranée. 